\title{
Parathyroid cyst: a rare case report
}

\author{
P. N. Sreeramulu • K. Borappa · S. L. Girish Gowda
}

Received: 1 March 2007 / Accepted: 4 January 2009

(C) Association of Surgeons of India 2009

\begin{abstract}
Parathyroid cysts are rare lesions arising in the neck and anterior mediastinum. Fewer than 250 cases have been published in the literature. Parathyroid cysts constitute only $0.6 \%$ of all thyroid and parathyroid lesions, but should be considered in the differential diagnosis of anterior neck masses, particularly in the presence of hypercalcaemia.
\end{abstract}

Keywords Parathyroid cysts $\cdot$ Neck mass $\cdot$ Functioning . Non functioning parathyroid cysts

\footnotetext{
P. N. Sreeramulu · K. Borappa · S. L. Girish Gowda Department of General Surgery, Sri Daveraj Urs Medical College (SDUMC), Kolar - 563 101, Karnataka, India

P. N. Sreeramulu ( $\square)$

E-mail: gowda1212@yahoo.co.in
}

\section{Case report}

A 60-year-old female patient presented with a left side neck mass present since 6 months (Fig. 1). She had no symptoms of dysphagia and dyspnoea. There was a tense cystic swelling present in relation to the left upper pole of the thyroid on examination. Swelling did move with

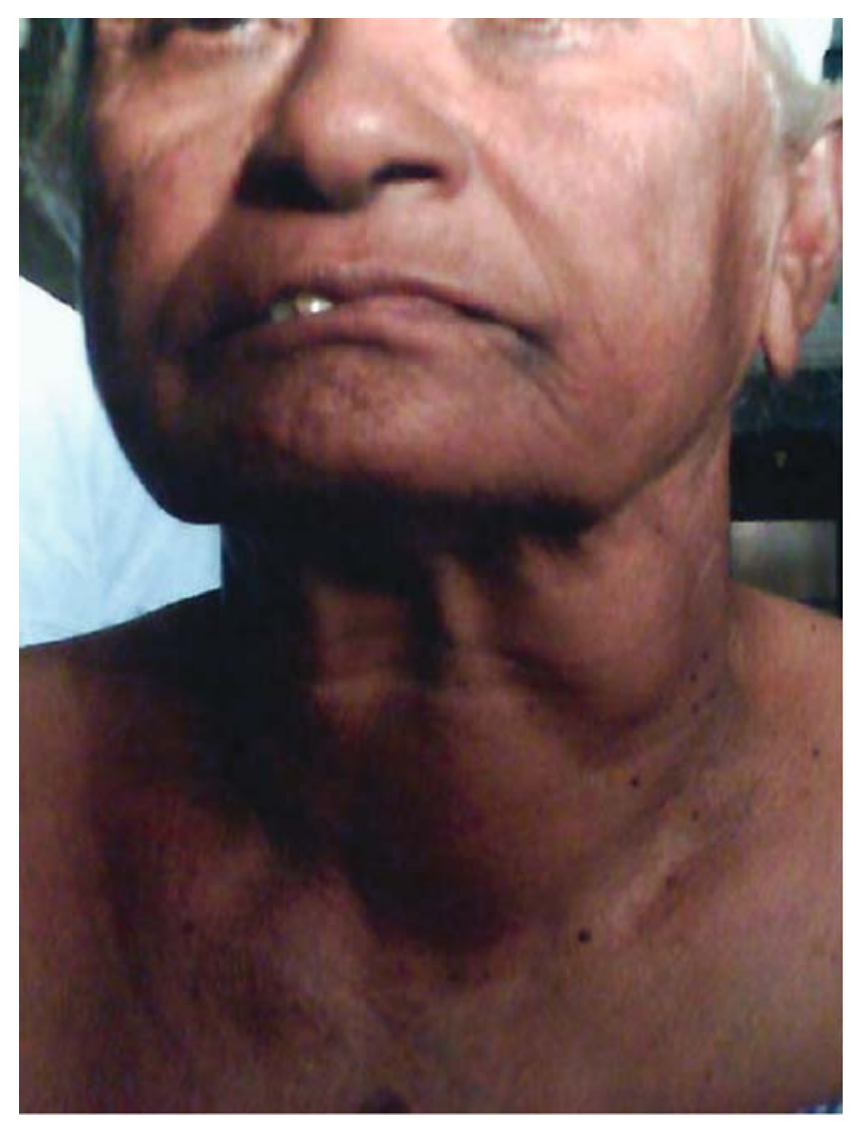

Fig. 1 Preoperative left side neck mass 
deglutination and swelling was mildly compressible. Patient was subjected for fine needle aspiration (FNA) under USG. Brownish fluid was aspirated and was not possible to define the origin of the lesion. USG of the neck revealed cystic lesion measuring $6.5 \times 4.5 \mathrm{~cm}$ in left lobe of thyroid without internal echoes inside the cyst. A provisional diagnosis of thyroid cyst was made. TSH, $\mathrm{T}_{4}, \mathrm{~T}_{3}$, serum calcium were all under normal limits. X-ray of the neck showed gross deviation of the trachea to right side.

Patient was subjected for surgery. A large cystic lesion measuring about $9 \times 5 \times 4 \mathrm{~cm}$ with thin wall was found arising from the upper pole of the left lobe of the thyroid (Fig. 2). Cyst was excised with the isthmus. Pre-op and postop period was uneventful. Histopathological examination revealed fibrous capsule surrounded by thyroid tissue. The cyst wall, along the luminal side, showed parathyroid cells in sheets and acinar pattern.

\section{Discussion}

Parathyroid cysts are rare lesions arising in the neck and anterior mediastinum. Fewer cases have been published in the literature [1]. The literature has focused on two broad categories of parathyroid cysts: functioning and

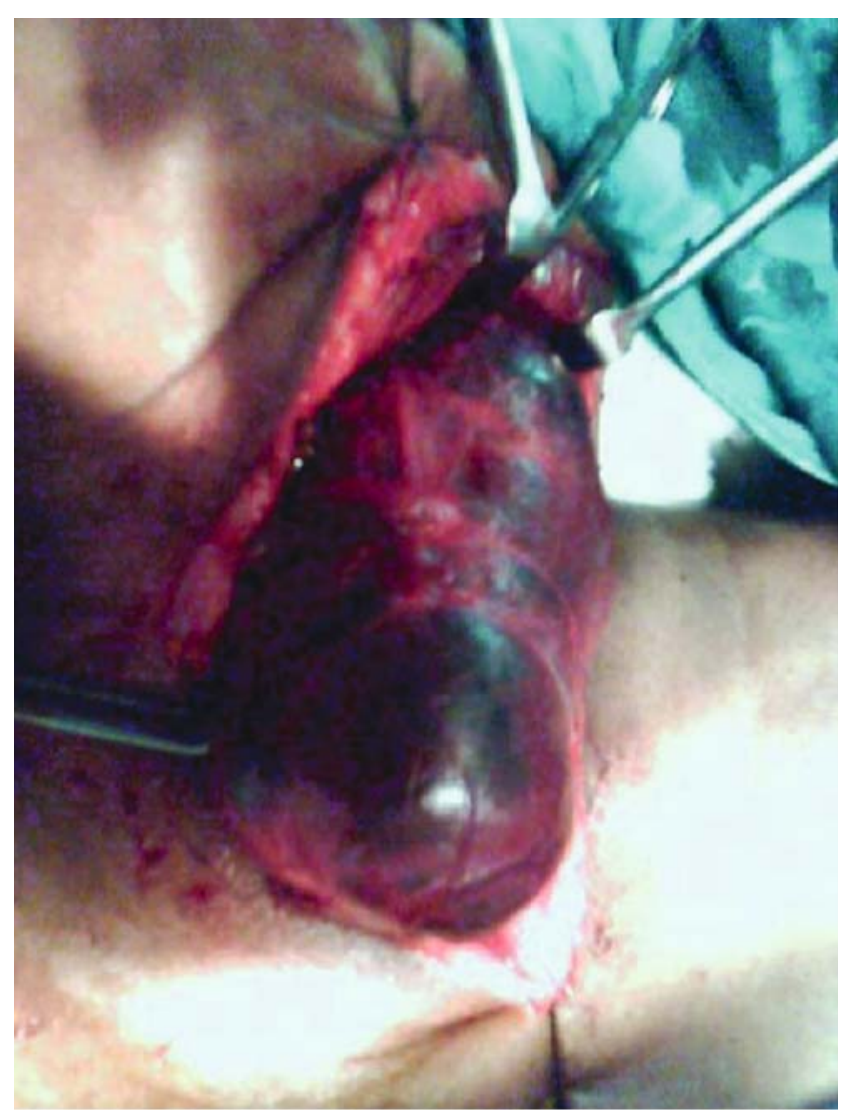

Fig. 2 Large cyst arising from the upper pole of the left lobe of the thyroid nonfunctioning, the former being associated with clinical hyperparathyroidism. The majority of patients, nearly $91 \%$, have nonfunctioning cysts [2]. In this group, women outnumber men 3 to 1 , and the mean age of affected individuals is 43.3 years. In contrast, men are more likely to have functioning cysts, outnumbering women 1.33 to 1 , and tend to be older with a mean age of 51.9 years [3]. The location of the cysts differs between the two groups. Nonfunctioning cysts are typically found in the inferior parathyroid and occur $60 \%$ of the time on the left side. Functioning cysts are much less predictable and have been located anywhere from the angle of the mandible to the mediastinum [4].

Aetiology of parathyroid cysts is uncertain, but 4 major theories suggest that they develop from embryological remnants of the third or fourth branchial pouch [5], coalescence of microcysts [6], simple retention of parathyroid secretions [7] or cystic degeneration in preexisting adenomas [8]. No single theory completely explains their origin, and many factors probably contribute to their aetiology.

To correctly diagnosis of a parathyroid cyst requires 3 steps. Consideration of parathyroid cysts in the differential diagnosis of a cystic mass in the neck, thyroid or elsewhere in the mediastinum, FNA and analysis of cyst fluid for parathyroid hormone (PTH). Although rare, parathyroid cysts are being detected more frequently due to widespread application of FNA, and it is particularly important to distinguish parathyroid from thyroid cysts. The former typically produce a clear, colourless fluid, whereas the latter contain fluid that is amber or brown. The presence of amber, brown, or even haemorrhagic fluid, however, does not exclude the diagnosis of parathyroid cyst.

After the diagnosis has been made, management depends upon whether the cyst is functioning or nonfunctioning. The definitive treatment for both remains surgical removal. This can be avoided, however, in nonfunctioning cysts by performing FNA. Complete disappearance of nonfunctioning cysts after FNA is well documented in the literature [9]. In one instance of a recurring nonfunctioning cyst, successful sclerosis was performed with injection of tetracycline, and surgery was avoided [10]. Observation without intervention may be appropriate in small, no symptomatic cysts. With functioning cysts, the consensus is that surgical removal is warranted. Although our review of the literature failed to find any reports of multiple functioning cysts, we recommend surgical exploration of all 4 parathyroid glands. Care should be taken to remove each encountered cyst or adenoma, leaving as many of the remaining parathyroid intact as is possible.

\section{Conclusions}

In conclusion, parathyroid cysts are rare, but the diagnosis should be entertained in a patient with a cystic neck mass, 
especially one occurring in the lower poles of the thyroid or discovered in a patient with hypercalcaemia. Diagnosis is best made by sending cyst fluid for PTH analysis. Serum calcium levels should be monitored after aspiration of suspected, functioning parathyroid cysts.

\section{References}

1. Pinney SP, Daly PA (1999) Parathyroid cyst: an uncommon cause of a palpable neck mass and hypercalcemia. West J Med 70:118-120

2. Layfield U (1991) Fine needle aspiration cytology of cystic parathyroid lesions: a cytomorphic overlap with cystic lesions of the thyroid. Acta Cytologica 35:447-450

3. Rosenberg 1, Orlando R, Ludwig M, Pyrtak U (1982) Parathyroid cysts. Am Surg 143:473-480
4. Waldron CA (1990) Face, lips, tongue, teeth, oral soft tissues, jaws, salivary glands and neck. In: Kissane M(Ed.): Anderson's Pathology. St. Louis, Mo, CV Mosby, pp 1095-1151

5. Troster M, Chiu HF, McLarty TO (1978) Parathyroid cysts: report of a case with ultrastructural observations. Surgery $83: 238-242$

6. Castleman B, Mallory TB (1935) The pathology of the parathyroid gland in hyper parathyroidism. Am Pathol 11: $1-80$

7. Selye H, Ortega MR, Tuchweber B (1964) Experimental production of parathyroid cysts. Am Pathol 45:251-259

8. Rogers LA, Finer B, Pene WB (1969) Parathyroid cyst and cystic degeneration of parathyroid adenoma. Arch Pathol 88:476-479

9. Ginsberg L, Young JEM, Walfish PG (1978) Parathyroid cysts. IAMA 240:1506-1507

10. Sanchez A, Careno H (1993) Treatment of a nonfunctioning parathyroid cyst with tetracycline injection. Head Neck $15: 263-265$ 